PROCEEDINGS OF THE

AMERICAN MATHEMATICAL SOCIETY

Volume 135, Number 9, September 2007, Pages 2683-2687

S 0002-9939(07)08835-1

Article electronically published on April 5, 2007

\title{
CHARACTERS OF ASSOCIATION SCHEMES CONTAINING A STRONGLY NORMAL CLOSED SUBSET OF PRIME INDEX
}

\author{
AKIHIDE HANAKI \\ (Communicated by Jim Haglund)
}

\begin{abstract}
We consider a relation between characters of an association scheme and its strongly normal closed subsets with prime index. As an application of our result, we show that an association scheme of prime square order with a proper strongly normal closed subset is commutative.
\end{abstract}

\section{INTRODUCTION}

It seems to be natural to consider relations between characters of an association scheme and its closed subsets. In [3], the author showed that Clifford theory works for commutative schemes and their strongly normal closed subsets very well. For non-commutative schemes, Clifford theory does not work so well. But for characters of association schemes and their strongly normal closed subsets, we can apply the theory of group-graded algebras by Dade [2].

Let $(X, S)$ be an association scheme in the sense of [11, and $T$ a strongly normal closed subset of $S$. For $x \in X$, the adjacency algebra of the subscheme $(X, S)_{x T}$, defined in [11, §1.5], is isomorphic to $\mathbb{C} T$. So we may regard $\mathbb{C} T$ as an adjacency algebra. In this article, we consider a relation between irreducible characters of $S$ and $T$, under the assumption that the factor scheme $(X, S)^{T}$, defined in [11, §1.5], is thin of prime order. Our result is a generalization of [9, Corollary 6.9] for group characters. But there are interesting cases which do not occur for group characters. We will construct such concrete examples. As an application, we will also show that $(X, S)$ is commutative if it has prime square order and there exists a proper strongly normal closed subset of $S$.

\section{Preliminaries}

Let $(X, S)$ be an association scheme. For $s \in S$, put $s^{*}=\{(y, x) \mid(x, y) \in s\}$. The valency of $s \in S$ will be denoted by $n_{s}$. Also, for a subset $U$ of $S$, put $n_{U}=\sum_{u \in U} n_{u}$. Especially, $n_{S}=|X|$, and this number will be called the order of $S$. For a closed subset $T$ of $S, n_{S} / n_{T}$ will be called the index of $T$ in $S$. The adjacency matrix of $s \in S$ is denoted by $\sigma_{s}$. For a subset $U$ of $S$, we put $\mathbb{C} U=\bigoplus_{u \in U} \mathbb{C} \sigma_{u}$

Received by the editors May 10, 2006.

2000 Mathematics Subject Classification. Primary 05E30.

Key words and phrases. Association scheme, strongly normal closed subset, character, Clifford theory, group-graded algebra.

(C)2007 American Mathematical Society Reverts to public domain 28 years from publication 
as a subset of the adjacency algebra $\mathbb{C} S$. It is known that the adjacency algebra over the complex number field $\mathbb{C}$ is a semisimple algebra. The set of irreducible complex characters of $S$ will be denoted by $\operatorname{Irr}(S)$. The map $\sigma_{s} \mapsto n_{s}$ is always an irreducible character of $S$. We call this the trivial character of $S$ and denote it by $1_{S}$. The central primitive idempotent corresponding to the trivial character is $n_{S}{ }^{-1} \sum_{s \in S} \sigma_{s}$. So $\sum_{s \in S} \sigma_{s}$ annihilates the irreducible $\mathbb{C} S$-module affording any non-trivial character.

Let $(X, S)$ be an association scheme, and $T$ a strongly normal closed subset of $S$. Put $G=S / / T$ and regard it as a finite group. We use Dade's notation in [2]. Then the adjacency algebra $\mathbb{C} S$ is a $G$-graded algebra with the grading

$$
\mathbb{C} S=\bigoplus_{s^{T} \in S / / T} \mathbb{C}(T s T)
$$

Let $\varphi$ be an irreducible character of $T$, and $L$ an irreducible right $\mathbb{C} T$-module affording $\varphi$. The induction of $L$ to $S$ is defined by

$$
L^{S}=L \otimes_{\mathbb{C} T} \mathbb{C} S=\bigoplus_{s^{T} \in S / / T} L \otimes \mathbb{C}(T s T) .
$$

Now $L \otimes \mathbb{C}(T s T)$ is a $\mathbb{C} T$-module for any $s \in S$. Define the support $\operatorname{Supp}\left(L^{S}\right)$ of $L^{S}$ by

$$
\operatorname{Supp}\left(L^{S}\right)=\left\{s^{T} \in S /|T| L \otimes \mathbb{C}(T s T) \neq 0\right\}
$$

and define the stabilizer $G\{L\}$ of $L$ in $G=S / / T$ by

$$
G\{L\}=\left\{s^{T} \in S / / T \mid L \otimes \mathbb{C}(T s T) \cong L \quad(\text { as } \mathbb{C} T \text {-modules })\right\} .
$$

Then $G\{L\}$ is a subgroup of $G$, but $\operatorname{Supp}\left(L^{S}\right)$ need not be a subgroup of $G$. In general, $\operatorname{Supp}\left(L^{S}\right)$ is a union of left cosets of $G\{L\}$ in $G$. It is shown in 2, Proposition 7.8] that $L \otimes \mathbb{C}(T s T)$ is an irreducible $\mathbb{C} T$-module for any $s^{T} \in \operatorname{Supp}\left(L^{S}\right)$.

We put $\operatorname{Supp}\left(\varphi^{S}\right)=\operatorname{Supp}\left(L^{S}\right)$ and $G\{\varphi\}=G\{L\}$. We write $\varphi^{s^{T}}$ for the character afforded by $L \otimes \mathbb{C}(T s T)$ for $s^{T} \in \operatorname{Supp}\left(\varphi^{S}\right)$.

For a character $\varphi$ of $T$ we write $\varphi^{S}$ for the induced character of $\varphi$ to $S$, and for a character $\chi$ of $S$ we write $\chi_{T}$ for the restriction of $\chi$ to $T$. We will use the following Frobenius reciprocity for characters of an association scheme and its closed subset. Let $\chi \in \operatorname{Irr}(S)$ and $\varphi \in \operatorname{Irr}(T)$. Then the multiplicity of $\chi$ in $\varphi^{S}$ is equal to the multiplicity of $\varphi$ in $\chi_{T}$. For example, this is shown by [10, Theorem 1.11.1] directly.

\section{Strongly nORmal Closed subsets of PRIME IndeX}

Let $(X, S)$ be an association scheme, and $T$ a strongly normal closed subset of $S$. Suppose $G=S / / T$ is isomorphic to the cyclic group of prime order $p$. Write $\operatorname{Irr}(G)=\left\{\xi_{i} \mid i=1,2, \cdots, p\right\}$. For $\chi \in \operatorname{Irr}(S)$, we define the product $\chi \xi_{i}$ by $\chi \xi_{i}\left(\sigma_{s}\right)=\chi\left(\sigma_{s}\right) \xi_{i}\left(\sigma_{s^{T}}\right)$. Then $\chi \xi_{i} \in \operatorname{Irr}(S)$ by [5].

Lemma 3.1. Let $\varphi$ be an irreducible character of $T$, and let $\chi$ be an irreducible constituent of $\varphi^{S}$. Suppose $\chi\left(\sigma_{s}\right) \neq 0$ for some $s \in S-T$. Then $\varphi^{S}=\sum_{i=1}^{p} \chi \xi_{i}$.

Proof. It is easy to see that $\chi \xi_{i}(i=1,2, \cdots, p)$ are all distinct. For every $i$, $\left(\chi \xi_{i}\right)_{T}=\chi_{T}$ contains $\varphi$ as an irreducible constituent. By the Frobenius reciprocity, $\varphi^{S}$ contains $\chi \xi_{i}$ for all $i$. Since $\left(\varphi^{S}\right)_{T}$ is a sum of at most $p$ irreducible characters, so is $\varphi^{S}$. This shows the lemma. 
Lemma 3.2. For $\varphi \in \operatorname{Irr}(T)$, suppose $G\{\varphi\}=G$. Then $\varphi^{S}=\sum_{i=1}^{p} \chi \xi_{i}$ for some $\chi \in \operatorname{Irr}(S)$ with $\chi_{T}=\varphi$.

Proof. Let $\left\{\chi_{j} \mid j=1,2, \cdots, r\right\}$ be the set of irreducible constituents of $\varphi^{S}$. If $\chi_{j}\left(\sigma_{s}\right) \neq 0$ for some $j$ and some $s \in S-T$, then Lemma 3.1 shows the lemma. So we may assume $\chi_{j}\left(\sigma_{s}\right)=0$ for every $j$ and every $s \in S-T$. Then $\left(\chi_{j}\right)_{T}=$ $\left(\chi_{j}(1) / \varphi(1)\right) \varphi$ shows that $\left\{\chi_{j} \mid j=1,2, \cdots, r\right\}$ is linearly dependent if $r>1$. But any subset of $\operatorname{Irr}(S)$ is linearly independent, so we have $r=1$. If $\varphi^{S}=a \chi_{1}$, then by Frobenius reciprocity we have $\left(\varphi^{S}\right)_{T}=a^{2} \varphi$. But we know that $\left(\varphi^{S}\right)_{T}=p \varphi$ by the assumption $G\{\varphi\}=G$. This is a contradiction.

Lemma 3.3. For $\varphi \in \operatorname{Irr}(T)$, suppose $G\{\varphi\}=1$. Then $\varphi^{S} \in \operatorname{Irr}(S)$. Put $\chi=\varphi^{S}$. Then $\chi\left(\sigma_{s}\right)=0$ for any $s \in S-T$ and $\chi_{T}$ is a sum of $\left|\operatorname{Supp}\left(\varphi^{S}\right)\right|$ distinct irreducible characters of T. For every irreducible constituent $\psi$ of $\chi_{T}$, we have $\psi^{S}=\chi$.

Proof. We have $\left(\varphi^{S}\right)_{T}=\sum_{s^{T} \in \operatorname{Supp}\left(\varphi^{S}\right)} \varphi^{s^{T}}$ and all $\varphi^{s^{T}}$ are distinct. If there exist an irreducible constituent $\chi$ of $\varphi^{S}$ and an element $s \in S-T$ such that $\chi\left(\sigma_{s}\right) \neq 0$, then Lemma 3.1 shows that $\left(\varphi^{S}\right)_{T}=p \varphi$. This is impossible, so we can say that $\chi\left(\sigma_{s}\right)=0$ for every $s \in S-T$ and every irreducible constituent $\chi$ of $\varphi^{S}$. By the same argument in the proof of Lemma 3.2, there exists only one irreducible constituent $\chi$ of $\varphi^{S}$. Since the multiplicity of $\varphi$ in $\left(\varphi^{S}\right)_{T}$ is one, we have $\varphi^{S}=\chi$. The rest of the statements in the lemma are clear by [2, $\S 7]$.

Since $G=S / / T$ is a cyclic group of prime order, $G\{\varphi\}=1$ or $G\{\varphi\}=G$ holds. Combining Lemmas 3.2 and 3.3, we have the following.

Theorem 3.4. Let $(X, S)$ be an association scheme, and $T$ a strongly normal closed subset of $S$. Suppose $G=S / / T$ is isomorphic to the cyclic group of prime order p. Write $\operatorname{Irr}(G)=\left\{\xi_{i} \mid i=1,2, \cdots, p\right\}$. Then, for $\chi \in \operatorname{Irr}(S)$, one of the following statements holds:

(1) $\chi_{T} \in \operatorname{Irr}(T)$ and $\left(\chi_{T}\right)^{S}=\sum_{i=1}^{p} \chi \xi_{i}$.

(2) $\chi\left(\sigma_{s}\right)=0$ for any $s \in S-T$ and $\chi_{T}$ is a sum of at most $p$ distinct irreducible characters. If $\psi$ is an irreducible constituent of $\chi_{T}$, then $\psi^{S}=\chi$.

Now we give examples of Theorem 3.4. The cases Theorem 3.4 (1) and (2) with $\operatorname{Supp}\left(\varphi^{S}\right)=G$ appear in the usual Clifford theory for finite groups. Theorem 3.4 (2) with $\operatorname{Supp}\left(\varphi^{S}\right)=1$ occurs for non-trivial characters of wreath products of association schemes [7]. We are interested in the case $G\{\varphi\}=1$ and $1 \subsetneq$ $\operatorname{Supp}\left(\varphi^{S}\right) \subsetneq G$. In this case, $\operatorname{Supp}\left(\varphi^{S}\right)$ is not a subgroup of $G$ since we are assuming that $G$ is a cyclic group of order $p$.

Example 3.5. Let $\mathbb{F}$ be the field with $2^{q}$ elements. To simplify our argument, we suppose $p=2^{q}-1$ is a prime number. The multiplicative group $\mathbb{F}^{\times}=\mathbb{F}-\{0\}$ acts on $\mathbb{F}$ by multiplication. Let $H$ be the semidirect product of the additive group $\mathbb{F}$ by $\mathbb{F}^{\times}$. Then $H$ is a Frobenius group with kernel $\mathbb{F}$. Let $K$ be a subgroup of $\mathbb{F}$ of order $2^{r}$. We can define a Schurian scheme $(X, S)$ by $H$ and its subgroup $K$. Namely $(X, S)$ is the factor scheme of the thin scheme defined by the group $H$ by its closed subset $K$. Let $T$ be the image of $\mathbb{F}$ in $S$. Then $T$ is a strongly normal closed subset of prime index $p$. Let $\chi$ be the unique nonlinear character of $H$. By [1, Theorem 11.25] or [6, Theorem 3.8], $\chi$ defines an irreducible character $\chi^{\prime}$ of $S$. Now we have $\left|\operatorname{Supp}\left(\varphi^{S}\right)\right|=\chi^{\prime}(1)=2^{q-r}-1$ for any irreducible constituent $\varphi$ of $\chi^{\prime} T$. So, if $1<q-r<q$, then $1 \subsetneq \operatorname{Supp}\left(\varphi^{S}\right) \subsetneq S / / T$. 


\section{Association schemes of PRIME SQUARE ORDER}

In [8, Theorem 3.3], it is shown that the following theorem holds.

Theorem 4.1 (Hanaki-Uno). Let $(X, S)$ be an association scheme. If the order of $(X, S)$ is a prime number, then $(X, S)$ is commutative. Moreover, all non-trivial irreducible characters of $S$ are algebraically conjugate.

It is natural to ask whether an association scheme $(X, S)$ of prime square order is commutative. No counterexample to the question is known. In this section, we will give a partial answer to this question as an application of the results in the previous section. Namely the following theorem holds.

Theorem 4.2. Let $p$ be a prime number, $(X, S)$ an association scheme of order $p^{2}$ which has a proper strongly normal closed subset $T$. Then $(X, S)$ is commutative. Moreover, if $n_{T}=p$, then $|S|=p|T|$ or $|S|=|T|+p-1$.

Proof. If $n_{T}=1$, then $S$ is thin and commutative.

We suppose $n_{T}=p$. Then we can apply Theorem 3.4. Note that $\mathbb{C} T$ is a commutative algebra by Theorem 4.1, so every irreducible character of $T$ is linear. For any $\chi \in \operatorname{Irr}(S)$, there exists $\varphi \in \operatorname{Irr}(T)$ such that $\varphi^{S}$ contains $\chi$ as an irreducible constituent. So it is enough to show that every irreducible constituent of $\varphi^{S}$ is linear for any $\varphi \in \operatorname{Irr}(T)$.

For the trivial character $1_{T}$ of $T$, every irreducible constituent of $\left(1_{T}\right)^{S}$ is linear since it is essentially a character of the cyclic group of order $p$.

Let $\varphi$ be a non-trivial irreducible character of $T$. We say that $\varphi$ is extendible to $S$ if there exists a character of $S$ whose restriction to $T$ is $\varphi$. If $\varphi$ is extendible to $S$, then every irreducible constituent of $\varphi^{S}$ is linear. Assume that $\varphi$ is not extendible to $S$. Put $\chi=\varphi^{S}$. Then $\chi \in \operatorname{Irr}(S)$ and $\chi(1)>1$. The number of non-trivial irreducible characters of $T$ is at most $p-1$, so we have $\chi(1)<p$. By Theorem 3.4 . we have $\chi\left(\sigma_{s}\right)=0$ for any $s \in S-T$.

For $s \in S-T$, we have that the valency $n_{s}$ of $s$ is at most $p$ by our assumption. In [4], it is show that $n_{s}$ is the unique eigenvalue of $\sigma_{s}$ in an arbitrary field of characteristic $p$. So we have

$$
0=\chi\left(\sigma_{s}\right) \equiv \chi(1) n_{s} \quad(\bmod p) .
$$

Therefore $n_{s}=p$ holds for any $s \in S-T$. This means that $T s T=\{s\}$. Note that the inverse of the group element $s^{T} \in S / / T$ is $\left(s^{*}\right)^{T}$. Then $\mathbb{C}(T s T) \mathbb{C}\left(T s^{*} T\right)=$ $\left(\mathbb{C} \sigma_{s}\right)\left(\mathbb{C} \sigma_{s^{*}}\right)=\mathbb{C}\left(\sum_{t \in T} \sigma_{t}\right)$, and this annihilates the irreducible $\mathbb{C} T$-module affording $\varphi$. Now [2, Theorem 8.6] shows that $s^{T} \notin \operatorname{Supp}\left(\varphi^{S}\right)$. So we have $\operatorname{Supp}\left(\varphi^{S}\right)=1$. This contradicts $\chi(1)>1$.

The trivial character $1_{T}$ of $T$ has exactly $p$ extensions to $S$. Any non-trivial irreducible character $\varphi$ of $T$ is extendible to $S$. Let $\chi$ be one of the extensions of $\varphi$. If $\chi\left(\sigma_{s}\right) \neq 0$ for some $s \in S-T$, then $\varphi$ has exactly $p$ extensions. Then every non-trivial irreducible character of $T$ has exactly $p$ extensions since they are algebraically conjugate. In this case, we have $|S|=p|T|$. If $\chi\left(\sigma_{s}\right)=0$ for any $s \in S-T$, then $\varphi$ has exactly one extension. Then every non-trivial irreducible character of $T$ has exactly one extension, and we have $|S|=|T|+p-1$.

\section{ACKNOWLEDGMENTS}

The author thanks M. Hirasaka, M. Muzychuk, P.-H. Zieschang, and the anonymous referee for their comments and suggestions. 


\section{REFERENCES}

1. C. W. Curtis and I. Reiner, Methods of representation theory. Vol. I, John Wiley \& Sons Inc., New York, 1981. MR0632548(82i:20001)

2. E. C. Dade, Clifford theory for group-graded rings, J. Reine Angew. Math. 369 (1986), 40-86. MR:0850628 (87m:16003)

3. A. Hanaki, Clifford theory for commutative association schemes, to appear in Discrete Math.

4. L Locality of a modular adjacency algebra of an association scheme of prime power order, Arch. Math. (Basel) 79 (2002), no. 3, 167-170. MR1933373 (2003h:05191)

5. Character products of association schemes, J. Algebra 283 (2005), no. 2, 596-603. MR:2111211 (2005m:05240)

6. A. Hanaki and M. Hirasaka, Theory of Hecke algebras to association schemes, SUT J. Math. 38 (2002), no. 1, 61-66. MR1919947 (2003g:05134)

7. A. Hanaki and K. Hirotsuka, Irreducible representations of wreath products of association schemes, J. Algebraic Combin. 18 (2003), no. 1, 47-52. MR2002219 (2004f:05193)

8. A. Hanaki and K. Uno, Algebraic structure of association schemes of prime order, J. Algebraic Combin. 23 (2006), no. 2, 189-195. MR2223686

9. I. M. Isaacs, Character theory of finite groups, Academic Press [Harcourt Brace Jovanovich Publishers], New York, 1976. MR0460423 (57:417)

10. H. Nagao and Y. Tsushima, Representations of finite groups, Academic Press Inc., Boston, MA, 1989. MR0998775 (90h:20008)

11. P.-H. Zieschang, An algebraic approach to association schemes, Lecture Notes in Mathematics, vol. 1628, Springer-Verlag, Berlin, 1996. MR1439253(98h:05185)

Faculty of Science, Shinshu University, Matsumoto, 390-8621, Japan

E-mail address: hanaki@math.shinshu-u.ac.jp 